\title{
HIGHLIGHTS
}

PAIN

\section{Do cytokines cause pain in small-fiber neuropathy?}

According to a case-control study published in Neurology, the expression of proinflammatory cytokines is elevated in the affected skin of patients with small-fiber neuropathy (SFN). "Our study is the first to investigate a possible pathophysiological role for local cytokine gene expression in this condition," comments lead author Nurcan Üçeyler, University of Würzburg, Germany.

\section{4 ...patients with SFN had} approximately twofold greater systemic gene expression of IL-2, IL-10 and transforming growth factor- $\beta 1 \ldots 77$

SFN can be associated with numerous metabolic disorders, and is characterized by spontaneous burning pain in the extremities, most commonly the feet. This disorder specifically affects A- $\delta$ and $C$ fibers, which are neurons that sense painful stimuli and convey information regarding noxious insults to the CNS. The underlying pathophysiological mechanisms that cause the debilitating burning pain associated with SFN are currently unknown.

Proinflammatory cytokines are typically algesic, and evidence suggests that these pleiotropic molecules might cause pain in various chronic pain conditions. To ascertain whether proinflammatory cytokines are involved in the genesis of pain in SFN, Üçeyler and colleagues quantified the cutaneous expression of cytokines in skin biopsy samples taken from the site of pain (distal calf) and at an unaffected site (proximal thigh). The researchers also analyzed blood samples taken from the patients to assess systemic cytokine gene expression.

"The most significant finding of our study was that in patients with lengthdependent SFN, the gene expression of the proinflammatory cytokines interleukin (IL)- 6 and IL-8 was significantly increased in the affected skin [compared with the unaffected skin and skin from healthy controls]," says Üçeyler. The researchers also found that patients with SFN had approximately twofold greater systemic gene expression of IL-2, IL- 10 and transforming growth factor- $\beta 1$ than had healthy controls.

Inflammatory cells are an important source of both anti-inflammatory and proinflammatory cytokines.

Nevertheless, the researchers found that macrophage and $\mathrm{T}$ lymphocyte numbers were similar between skin biopsy samples taken from affected and unaffected skin and between patients with SFN and controls. This result indicates that the increased cytokine expression in affected skin was not associated with the degree of inflammatory cell infiltration.

The results of the Üçeyler et al. study indicate that $A-\delta$ and $C$ fibers that are damaged in SFN might be sensitive to algogens, and elevated local proinflammatory cytokines might be one of the underlying causes of pain associated with this condition. However, "future studies with higher numbers of patients and controls are needed to confirm and extend our findings," write the authors.

The researchers now plan to use organ culture techniques to identify the cellular source of the elevated local cytokines.

Nick Jones

Original article Üçeyler, N. et al. Elevated proinflammatory cytokine expression in affected skin in small fiber neuropathy. Neurology 74, 1806-1813 (2010) 